URINARY TRACT INFECTION OF CHILDREN: ETIOLOGIC AGENTS AND THEIR RESISTANCE PATHERNS

\author{
A. Mahyar ${ }^{1}$, S. Mahyar ${ }^{2}$ \\ ${ }^{1}$ Pediatrics, ${ }^{2}$ Pharmacy, Qazvin University of \\ Medical Sciences, Qazvin, Iran
}

Background: Urinary tract infection is common disease in children. Delay in appropriate diagnosis and treatment results in severe complications. Identification of etiologic agents and resistance pathern of bacterial microorganisms is very important.

Objective: To determine the etiologic agents and resistance patherns in urinary tract infection of children.

Methods and materials: This cross -sectional study has been conducted on 74 hospitalized children affected with urinary tract infection in Qazvin children hospital affiliated to Qazvin University of Medical Sciences, Qazvin (Iran) in 2007. All of cases had positive urine culture $>10^{5} \mathrm{CFU} / \mathrm{ml}$ of a given organism. The culture performed using standard methods. Data was analyzed by using statistical methods.

Results: Out of 74 children affected with urinary tract infection 10(\%13.5) were male and 64(\%86.5) females. The mean age was $3 \pm 1.8$ years. The most common causative agents were Escherichia coli 61(\%82.2) and Klebsiella 11(14.8). Resistance to Antibiotics in all isolates were : ampicillin 49 (66.3\%) , co-trimoxazole 47 (63.5\%) ,cephalothin 35(47.3\% ), nalidixic acid 33( 44.6\%), ceftriaxone31(41.9\% ), amikacin17(21.9 ), nitrofurantoin 11(14.9\% ), gentamycin $9(22.1 \%)$,ciprofloxacin $5(6.6 \%)$ and cefazolin $1(1.3 \%)$.

Conclusion: This study revealed that Escherichia coli was the most common agent.The highest antimicrobial resistance was observed for ampicillin and co-trimoxazole.

\section{TO DO OR NOT TO DO: WHEN THE LATTER IS BETTER. A COHORT STUDY ABOUT NICE GUIDELINES IN UTI}

\author{
M. Pennesi, L. Travan, I. L'Erario
}

Dep of Pediatrics, Institute for Child and Maternal Health, IRCCS Burlo Garofolo, Trieste, Italy

Background and aims: The guidelines from the National Institute for Health and Clinical Excellence (NICE 2007) on urinary tract infections (UTI) in childhood led to an epochal change in the management of children with UTI decreasing the number of instrumental evaluations. We used a protocol almost identical to that proposed by NICE. The aim of the study was to describe the 11 years follow-up of children with UTI and to validate our protocols.

Methods: We enrolled 406 children (1-36 months) at their first UTI. All the children underwent renal ultrasounds (US). Children with pathological US and those with UTI recurrences underwent voiding cystourethrography (VCUG)and dimercaptosuccinic acid (DMSA) renal scans. All of them were clinically followed up.

Results: US after the first episode of UTI were normal in 376 children (92.6\%), pathological in 30 (7.4\%). 18 patients had a second episode of UTI $(4.4 \%)$. Second renal US were normal in all of them. We performed 48 VCUG: 30 in children with pathological US and 18 because of UTI recurrences. 14 children had VUR (29\%): 12 had a pathological ultrasound and 2 had recurrent UTIs. The same children underwent DMSA scans: renal damage appeared only in 6 children $(12.5 \%)$; all these children showed VUR of grade IV. The remaining 42 did not show any damage.

Conclusions: Our study shows that application of NICE guidelines leads to a significant saving of invasive evaluations without compromising child health. Moreover our data confirm that is unreasonable searching VUR since the first episode of UTI. 